\title{
Simultaneous scanning ion conductance and atomic force microscopy with a nanopore: Effect of the aperture edge on the ion current images
}

\author{
Livie Dorwling-Carter, ${ }^{1}$ Morteza Aramesh, ${ }^{1}{ }^{1}$ a) Csaba Forró, ${ }^{1}$ Raphael F. Tiefenauer, ${ }^{1}$ \\ Ivan Shorubalko, ${ }^{2}$ János Vörös, ${ }^{1}$ and Tomaso Zambelli ${ }^{1, a)}$ \\ ${ }^{1}$ Laboratory of Biosensors and Bioelectronics, ETH Zurich, Gloriastrasse 35, Zurich 8092, Switzerland \\ ${ }^{2}$ Laboratory for Transport at Nanoscale Interfaces, Swiss Federal Laboratories for Materials Science and \\ Technology (Empa), Ueberlandstrasse 129, Dübendorf 8600, Switzerland
}

(Received 27 August 2018; accepted 14 October 2018; published online 1 November 2018)

\begin{abstract}
Scanning ion conductance microscopy (SICM) is a technique for high-resolution non-contact imaging, particularly powerful for live cell studies. Despite debates on its lateral resolution, consensus is that a probe presenting a tip with small opening aperture, large opening angle, and large outer-to-inner radius ratio will offer a SICM current signal more sensitive to tip-sample separation, ultimately impacting the image resolution. We report here the design of such a probe, integrating a nano-opening $(<20 \mathrm{~nm}$ opening diameter) with increased outer-to-inner radius ratio and a wide opening angle through microfabrication and ion milling. The probe consists of a microfluidic atomic force microscopy (AFM) cantilever offered by the Fluid Force Microscope (FluidFM) technology, able to act as an SICM and AFM probe. Such a combination allows investigating the implications of the new probe geometry on the SICM imaging process by simultaneously recording currents and forces. We demonstrate through experiments on well-defined samples as well as corresponding simulations that by integrating a nanopore onto the FluidFM, nanoscale features could be successfully imaged, but the increased sensitivity of the probe current to sample distance comes with higher sensitivity to an inherent SICM wall artefact. Published by AIP Publishing.

https://doi.org/10.1063/1.5053879
\end{abstract}

\section{INTRODUCTION}

Scanning ion conductance microscopy ${ }^{1,2}$ (SICM) has become an established scanning imaging technique at the nano and microscale. In particular, the non-contact nature of SICM turned this technology into a formidable imaging tool of live cells, ${ }^{3}$ in their physiological environment, at a higher resolution than atomic force microscopy $\left(\mathrm{AFM}^{4}\right)$ because of the absence of mechanical coupling artefacts or sample damage, ${ }^{5,6}$ Although AFM relies on attractive and repulsive forces between the sample and the apex of a probe to perform sample imaging, SICM uses an ion current generated between an electrode inside an electrolyte-filled probe and an electrode in bulk solution. When the probe approaches the sample, the hindrance of the ion flow into the tip leads to a drop in current that can be utilized as a feedback to accurately position the probe with respect to the sample and thus map the sample topography.

SICM resolution is impacted by several parameters that make its theoretical quantification subject to controversy. ${ }^{7-10}$ For example, Rheinlaender and Schäffer determined via finite element method (FEM) modeling ${ }^{7}$ and experiments ${ }^{10}$ a lateral resolution of $3 r_{i}$, where $r_{i}$ is the probe inner opening radius. The lateral resolution was defined as the center-to-center distance at which two small cylindrical particles could clearly be resolved from each other, i.e., with the existence of an imaged "dip" in between the closely spaced features. They also evidenced how the topographical features

\footnotetext{
a) Authors to whom correspondence should be addressed: aramesh@biomed. ee.ethz.ch and zambelli@biomed.ee.ethz.ch
}

can appear as bell-shaped or ring-shaped depending on their size and the working imaging distance. The $3 r_{i}$ lateral smearing of the imaged particles (full width at half-minimum, fwhm) further indicated the finite resolution of SICM, agreeing with the simulated results of Edwards et al. ${ }^{11}$ Del Linz et $a l .^{8}$ determined computationally this limit to $2 r_{i}$ for elongated objects (edge-to-edge feature distance), corresponding to a $4 r_{i}$ center-to-center distance. The features were considered resolved when the dip in height between them was $\geq 0.05 r_{i}$. This larger resolution than the $3 r_{i}$ estimated by Rheinlaender and Schäffer was associated to the wider features $^{10}$ simulated by Del Linz et al. On the other hand, Weber and Baker ${ }^{9}$ experimentally estimated a lateral resolution limit at $0.5 r_{i}$ (edge-to-edge feature distance) by imaging well-defined topography trenches fabricated by a focused ion beam (FIB). In this study, the resolution limit was determined by the possibility for the probe to sense the dip between two closely spaced features when the intensity in the interspace decreased $\geq 26 \%$ of the object height. With such a criterion, they experimentally found a lateral resolution as low as $0.5 r_{i}$, in agreement with other experimental results that were able to image features spaced by distances $\sim 0.5 r_{i}{ }^{12}$

Although quantification remains unsolved, the experimental and simulation works aiming to clarify SICM image interpretation agree that the following parameters are expected to influence the resolution: the probe aperture size, its working distance to the sample surface, its opening angle, the ratio $R$ of the tip outer radius $\left(r_{o}\right)$ to the inner radius $\left(r_{i}\right)$ $\left[R=r_{o} / r_{i}\right.$, thus depending on the probe wall thickness $\left(r_{o}-r_{i}\right)$, and finally the sample topography itself. ${ }^{7-11} \mathrm{~A}$ 
smaller probe aperture ensures a closer working distance and a higher current-distance sensitivity ${ }^{13}$ (defined as the current amplitude variations associated with a change of probesample distance, used for SICM feedback). A larger $R$ allows an increased squeezing of the current under the lateral walls of the probe. ${ }^{7,11}$ A larger opening angle reduces the probe internal resistance and as such favors higher distancedependent current amplitude variations: this originates from the fact that the total electrical resistance is a summation of the probe internal resistance with the outside resistance (including the distance-dependent resistance), mounted in series. Therefore, the lower the probe internal resistance, the higher the weight given to the distance-dependent resistance. ${ }^{7,11}$ The afore-mentioned factors (smaller openings, larger $R$, and probe opening angle) will consequently contribute to increasing the probe current-distance sensitivity (i.e., SICM sensitivity), ultimately affecting the resolution.

In this work, our interest was to improve the smallest topographical feature size (width) that can be detected by the probe current and the resulting SICM image when increasing the probe SICM sensitivity. For this, we have milled a nanopore at the apex of the pyramid of a microchannelled AFM cantilever [Fluid Force Microscope (FluidFM) ${ }^{14,15}$ Figs. 1(a) and $1(\mathrm{c})]$. The almost circular nanopore has a diameter of around $20 \mathrm{~nm}$ and offers thick lateral walls $(R>10)$ for increased ion constriction (i.e., "squeezing" between walls and sample). The pore opens on a wide opening angle pyramidal probe (half opening angle $\sim 35.26^{\circ}$ compared to conventional glass pipette angles of $\sim 2^{\circ}-4^{\circ}$ ) for reduced probe electrical resistance, all in agreement with factors previously identified as enhancing the SICM sensitivity. The nanopore being drilled on a FluidFM cantilever allows for simultaneous complementary force recording which helps to evidence eventual mechanical contacts with the sample. SICM has recently been demonstrated on FluidFM probes ${ }^{16}$ having a $300 \mathrm{~nm}$ opening diameter [Fig. 1(b), apex probe]. Here, we report the resolution improvement gained when customizing the probe to the previously mentioned nanopore. Our results show that unlike the apex probes, the scanning nanopore is indeed able to image features at the nanoscale (i.e., below $100 \mathrm{~nm}$ here), down to at least $65 \mathrm{~nm}$-wide features with sensitive current detection, but accompanied with an unavoidable image distortion due to the probe lateral dimensions (opening angle and lateral wall thickness). We especially reveal through experiments and FEM simulations a SICM border artefact on feature steps. The artefact is the result of the probe's walls and is therefore inherent to the SICM image formation process. This is of particular interest for works driving for higher resolution imaging by reducing the pore opening without adjusting the probe wall dimensions. The complementary force information provided by the FluidFM cantilever also proves its benefits for not only monitoring sample contacts but also interpreting the SICM imaging process.

\section{EXPERIMENTS AND MODELING}

\section{A. Solution}

A $150 \mathrm{mM} \mathrm{KCl} \mathrm{(Sigma-Aldrich)} \mathrm{electrolyte} \mathrm{solution}$ was used as the imaging solution and was prepared with ultrapure Milli-Q water (Millipore Corp., resistivity ca. 18.2 $\mathrm{M} \Omega \mathrm{cm}$ at $25^{\circ} \mathrm{C}$ ). The FluidFM probes were filled with the same solution as that of the bulk.

\section{B. Substrates}

\section{PDMS calibration grid}

The polydimethylsiloxane (PDMS, Sylgard 184, Dow Corning) structure was prepared according to the protocol described elsewhere. ${ }^{16}$ PDMS was poured and baked on the silicon calibration grid TGXYZ03 (Mikromasch Europe, Germany). The resulting patterned structure was cut out of the rest of the PDMS matrix and let spontaneously adhere on a plasma-cleaned glass Willco dish.

\section{Nanoprinted gold lines}

The nanoscale gold lines were prepared by template-stripping based nanotransfer printing (TS-nTP). ${ }^{17,18}$ A $10 \mathrm{~nm}$ gold layer was evaporated onto a template with nanoscale lines fabricated by extreme UV interference
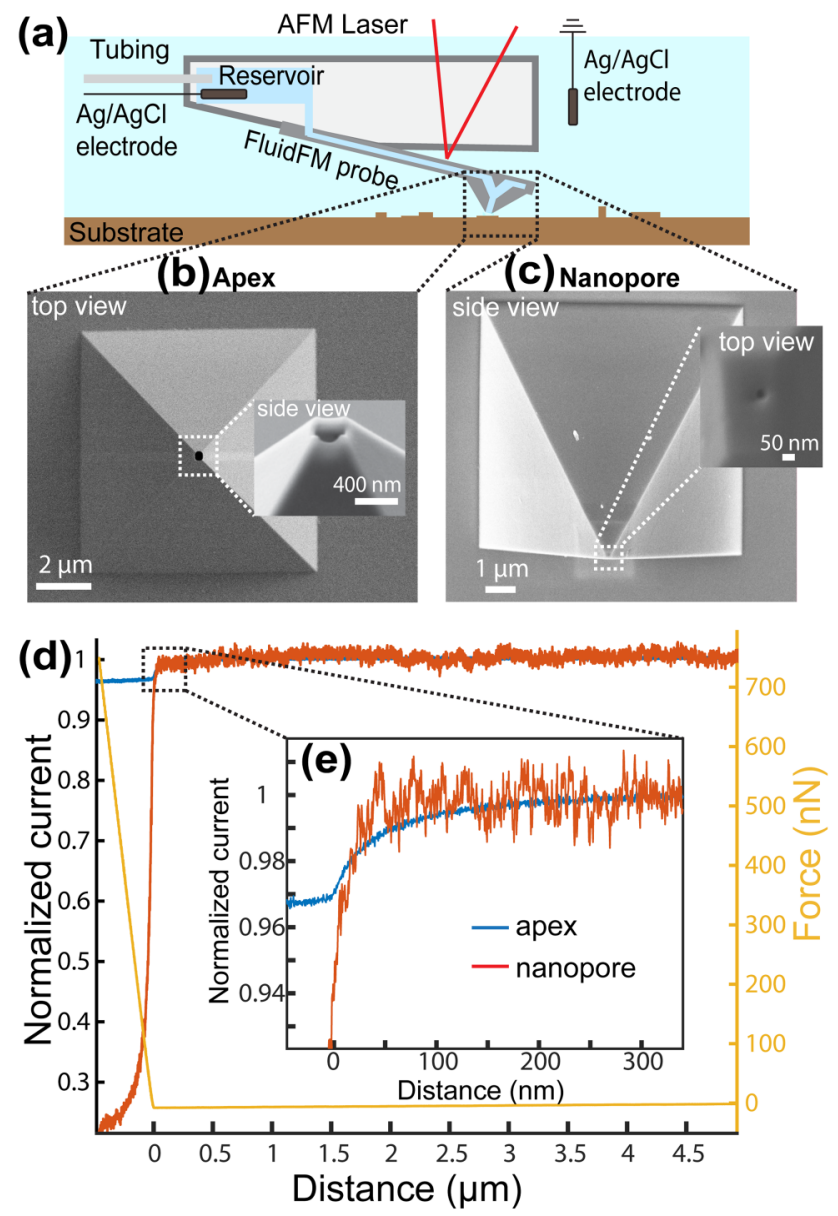

FIG. 1. (a) Schematic representation of a FluidFM probe used for simultaneous force and ion current imaging. (b) SEM images of a $300 \mathrm{~nm}$ opening diameter apex probe from top and side view. (c) SEM image (side view) and HIM image (top view) of an $18 \mathrm{~nm}$-opening diameter nanopore probe. (d) Approach curves onto a glass substrate with an apex probe (blue curve) and the nanopore probe (red curve). The simultaneous force curve (orange, right axis) is used to identify the moment of contact of the probe with the substrate. The current is normalized to the ion current value in bulk solution. (e) Zoom into the approach curves close to contact with the sample. 
lithography. The topmost surface layer was stripped at $120^{\circ} \mathrm{C}$ by an adhesive PEN-supported PVA sheet (PEN: Teonex Q $125 \mu \mathrm{m}$, Synflex, Germany; PVA: Mowiol 18-88, Sigma Aldrich, Switzerland) and then transferred onto a glass slide at $120^{\circ} \mathrm{C}$ applying a manual pressure. The PEN support was subsequently peeled-off, and the PVA was dissolved in boiling water twice for $5 \mathrm{~min}$.

\section{Probes}

Commercially available FluidFM apex probes (opening diameter $300 \mathrm{~nm}$, stiffness of $2 \mathrm{~N} \mathrm{~m}^{-1}$ ) were purchased from Cytosurge (Switzerland). Nanopore FluidFM probes were customized from FluidFM probes without aperture ordered from Cytosurge and subsequently carbon-coated (CCU-010 $\mathrm{HV}$, safematic, Switzerland) for SEM-FIB manipulations. Their apex was milled by a Focused Ion Beam (FIB at 10 pA, NVision40, Carl Zeiss AG, Jena, Germany). The process was monitored by simultaneous SEM inspection and manually stopped just at the moment when the inner microchannel of the probe started to appear. Helium Ion Microscope (HIM, Zeiss Orion Plus) was used to fabricate the nanopores on the probes, offering lower sputtering rate for higher resolution tuning of the probe opening size. ${ }^{19,20}$ HIM was operated at $30 \mathrm{kV}$ acceleration voltage and $10 \mathrm{pA}$ ion beam current. Varying single pixel exposure time between 5 and $30 \mathrm{~s}$ led to opening diameters between 15 and $30 \mathrm{~nm}^{21}$ The probes were then cleaned with an air plasma for $8 \mathrm{~min}$ to remove the carbon layer and glued onto the standard microfluidics AFM holder (Cytoclip, Cytosurge). Before filling them with the $\mathrm{KCl}$ electrolyte, all the probes were plasma-cleaned for $2 \mathrm{~min}$ to increase the wettability of the microchannel. Probe filling was performed in a carbon dioxide saturated box to ease the process. In the case of the nanopore probe, a $30 \mathrm{~min}$ time was allowed before experimenting to ensure the full wetting of the nanopore opening from both sides.

\section{Instrumentation}

The FluidFM microscope (Cytosurge and Nanosurf AG, Switzerland) was adapted for ion conductance measurements by incorporating two $\mathrm{Ag} / \mathrm{AgCl}$ quasi-reference counter electrodes. ${ }^{16,22}$ An electrode was inserted in the reservoir of the probe (connected to a Pico 2 USB-powered patch clamp amplifier, Tecella, USA) and the other one in the bulk solution (connected to the amplifier's ground). The voltage bias was applied with the WinWCP software (University of Strathclyde, UK) while data were acquired with the Nanosurf's C3000 control software. All potential values reported in this work refer to the potential of the electrode in the reservoir of the FluidFM probe with respect to the electrode in the bulk solution.

\section{E. Experimental procedures}

Approach curves were recorded by moving the probe toward the glass substrate at a speed of $500 \mathrm{~nm} / \mathrm{s}$ and using a sampling rate of $1.17 \mathrm{kHz}$ with AFM force feedback. The force feedback was used to identify each moment of contact of the probe with the substrate. Images were acquired using raster scanning, at $45^{\circ}$ with respect to the cantilever axis, either in SICM mode (feedback on drop in current, relative to the bulk current) or in AFM contact mode (feedback on the cantilever deflection). The corresponding setpoints (SPs) are indicated for each experimental dataset. All images consisted of $256 \times 256$ pixels.

\section{F. Modeling}

A 2D model inspired by Rheinlaender and Schäffer $^{7}$ was conceived using COMSOL Multiphysics version 5.3 Electric Currents and Electrical Circuit modules. Two geometries have been investigated: a FluidFM nanopore probe reproducing the geometry as derived from the SEM images, and a nanopore of the same opening at the apex of a standard glass pipette. The gold feature (nanoline) to image is represented as a rectangle of varying width, centered at $\mathrm{x}=0 \mu \mathrm{m}$. The image in current $I(x)$ is simulated by laterally displacing the probe along the $\mathrm{x}$ axis, at a constant defined vertical probe position. During experimental imaging, the probe is vertically positioned at each $\mathrm{x}$ position by an AFM force feedback ensuring constant applied forces. Mimicking this experimental positioning process to estimate, for each $\mathrm{x}$, the vertical probe position that would match the force setpoint would be too computationally intensive. Instead, positioning the tip at a constant vertical position above the glass sample at a distance calculated from the probe geometry (Supporting Section SI-6 of the supplementary material) allows simulating the imaging process. Indeed, this recreates the local increased working distance of the probe (relatively to the reduced distance on top of the feature) when the probe is laterally distant from the gold feature (due to the tip "AFM" convolution observed experimentally). The simulated scan of a gold line was therefore carried out by parametric sweeping on the lateral position $\mathrm{x}$ of the probe while the vertical position was fixed. Such a strategy allows simplifying the computational time while reproducing the experimental observations.

\section{RESULTS AND DISCUSSION}

\section{A. Current-distance behavior on a planar surface}

The SICM and AFM imaging capabilities of the FluidFM are determined by the geometry and electrical properties of the scanning probe. The probes used in this manuscript consist of a commercially available apex probe with a $300 \mathrm{~nm}$ diameter opening [Fig. 1(b)] and of a custom probe with a nanopore fabricated by FIB and HIM milling [Fig. 1(c)]. The apex probe is used for comparison of the SICM results with the designed nanopore.

The apex and nanopore probes both consist of a hollow FluidFM cantilever linking a liquid reservoir to a pyramidal probe opening via a microfluidic microchannel [Fig. 1(a)]. The difference in the two probe geometries lies at the probe tip. The apex probe [Fig. 1(b)] consists of a $300 \mathrm{~nm}$-diameter etched hole, with four prominent apexes and a thin lateral wall thickness [ $\mathrm{ca} .40 \mathrm{~nm}$ estimated from SEM observation of Fig. 1(b), corresponding to a ratio $R \sim 1.1$ ], whereas the nanopore probe [Fig. $1(\mathrm{c})]$ is characterized by a smaller pore 
dimension better visible under a HIM than SEM. The HIM image reveals a pore opening diameter of $c a .18 \pm 2 \mathrm{~nm}$ and probe lateral wall dimensions of $c a$. average $100 \mathrm{~nm}$ (corresponding to $R \sim 12$ ). We derived its height from its measured probe electrical resistance of $47 \mathrm{M} \Omega$ in $150 \mathrm{mM} \mathrm{KCl}$ (Fig. S1 of the supplementary material). Assuming a cylindrical geometry for the nanopore connecting the external bulk environment with the hollow space of the FluidFM pyramid, the measured resistance leads to an estimated nanopore cylindrical height below $10 \mathrm{~nm}$. The small nanopore height is in stark contrast with the elongated geometry of the pulled glass pores, which is responsible for the several-fold higher probe electrical resistances at equivalent nanoscale diameter due to their limited opening angle. The reduced probe electrical resistance provides the FluidFM nanopore a higher sensitivity to the external resistances in series with the probe, including the distance-dependent resistance, not dominated by a prohibitive internal probe resistance ${ }^{11}$ (increased current amplitude variations).

To assess the sensitivity of the nanopore tip response to sample distance compared to an apex probe, two approach curves on glass were performed and are presented in Figs. 1(d) and 1(e). Glass was chosen as a flat and nondeformable substrate enabling to determine differences in the approach curves without sample artefact. The simultaneously recorded forces from the AFM feedback (orange curve) are used to determine the point of contact of the probes with the sample. Both approach curves are normalized to the current in bulk, herein at a probe distance of $5 \mu \mathrm{m}$. The corresponding bulk amplitudes are $1.812 \mathrm{nA}$ (standard deviation of $1.0 \mathrm{pA}$, probe electrical resistance $27 \mathrm{M} \Omega$ ) for the apex probe vs. $1.072 \mathrm{nA}$ (standard deviation of $5.7 \mathrm{pA}$, probe electrical resistance $47 \mathrm{M} \Omega$ ) for an applied bias of $50 \mathrm{mV}$. For all experiments, the bias applied at the probe corresponds to the potential applied at the electrode in the reservoir of the FluidFM with respect to the electrode in the bulk solution. With decreasing distances to the surface, both apex and nanopore current curves show a drop from the hindrance of the ion flow through the probe opening. The nanopore probe leads to a current drop down to 0.24 after contact, while the apex probe only drops to 0.966 . This important discrepancy originates from the different probe geometries: while the apex probe brings in contact a relatively large $300 \mathrm{~nm}$ opening that cannot get fully closed from its pointy apex geometry and limited $R$, the nanopore offers a reduced hole with considerable lateral thickness which can better constrain the ion flow as hindrance happens mostly under the probe walls. $^{7}$ A zoom into the pre-contact distances with the sample [Fig. 1(e)] shows the steeper drop in current achieved with the nanopore probe, indicative of a higher current sensitivity (a small change in distance is associated with a large change in current amplitude). Correlated with the steeper drop is the reduced distance range of sensitivity of the nanopore compared to the apex probe: for a conventional $1 \%$ current setpoint (SP) corresponding to a $1 \%$ drop in current amplitude relatively to the bulk, the working distance of the nanopore probe from the substrate is only $23 \mathrm{~nm}$ compared to $70 \mathrm{~nm}$ with the apex probe. The working distances could accurately be measured thanks to the FluidFM force feedback identifying the sample surface. The force feedback will similarly enable the recognition of the tip-sample mechanical contacts during SICM scanning of the imaging tip.

\section{B. Microscale and nanoscale SICM imaging}

The ability of the nanopore probe for simultaneous SICM imaging and force recording was investigated first by imaging a PDMS calibration grid of microscale dimensions [ $5 \mu \mathrm{m}$ wide features separated by $5 \mu \mathrm{m}$ as showed in the optical image of the scanned PDMS area of Fig. S2(a) of the supplementary material]. Figure 2(a) shows the resolved SICM topography by the FluidFM nanopore, imaging square features of $5 \mu \mathrm{m}$ and $450 \mathrm{~nm}$ height, close to the nominal values. The image was acquired with a continuous decrease in current SP, from an initial SP $=5.5 \%$ drop at the bottom of the image to a final drop of $\mathrm{SP}=2.75 \%$ at the end. This $2.75 \%$ SP was found to be at the limit of the probe SICM sensitivity as proved by the white lines that momentarily appear in the topography image: those lines correspond to an imaged constant topography since the SICM probe could not sense variations of topography due to the too far working distances. Such continuous SP adjustment, affecting the resolution of the features as seen in Fig. 2(a), was performed to optimize the SICM SP offering sufficient resolution while minimizing the continuously monitored forces onto the sample [Fig. 2(b)]. The simultaneous force map of Fig. 2(b) shows the still consequent forces applied on the sample at this optimal $2.75 \% \mathrm{SP}$, as the forces reach $128 \mathrm{nN}$, while they may increase up to $305 \mathrm{nN}$ at the initial higher SP of $5.5 \%$. The smaller range of tip-sample distances where the current of the nanopore probe is SICM sensitive [as identified in the approach curve of Fig. 1(e)] requires closer probe working distances, which translates in unavoidable contacts with the high PDMS step sample $(500 \mathrm{~nm}$ aspect-ratio nominally). On the contrary, the increased working distance offered by the apex probe allows non-contact imaging but at the cost of a lower resolution [as documented in Figs. S3(a) and S3(b) of the supplementary material]. The simultaneous forces therefore indicate the contacts that the nanopore probe exerts on the sample, which would not be identifiable on the SICM topography image, in agreement with the limit of $10 r_{i}$ maximum sample height simulated by Del Linz et al. ${ }^{8}$ for non-contact imaging.

We have just proved that the nanopore probe is able to perform SICM imaging of features at the microscale. As a next step, we tested its aptness to image nanoscale details. A sample consisting of patterned $10 \mathrm{~nm}$ high gold lines with varying dimensions (from 115 to $65 \mathrm{~nm}$ width, with a 300 $\mathrm{nm}$ pitch) was fabricated on glass via the recently-developed technique of template-stripping combined with nanotransfer printing. ${ }^{17}$ This technique allows via a patterned mold to deposit stable nanoscale features on a large substrate, advantageous for targeting the features to image with the probe [Fig S2(b) of the supplementary material]. Such a patterned sample was chosen for our study to image controlled features of known geometry and dimensions helping the interpretation of the obtained images. 

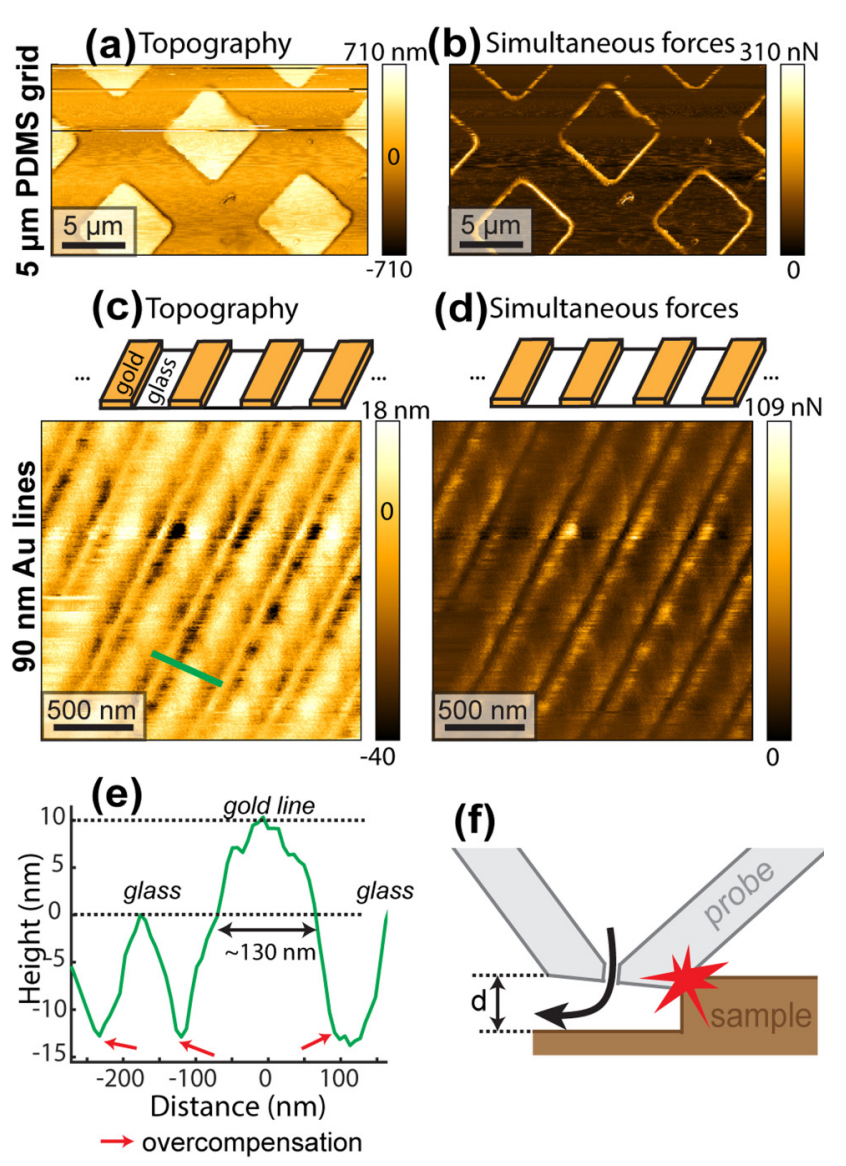

FIG. 2. (a) SICM topography and (b) simultaneous force recording when imaging with the nanopore probe a PDMS grid with $5 \mu \mathrm{m}$ wide squares separated by $5 \mu \mathrm{m}$ [SP from $5.5 \%$ drop (bottom) to $2.75 \%$ (top), bias $=90 \mathrm{mV}$ ]. (c) SICM topography and (d) simultaneous force recording when imaging with the nanopore probe nanoscale lines of $90 \mathrm{~nm}$ width, separated by 210 $\mathrm{nm}(300 \mathrm{~nm}$ pitch $)(\mathrm{SP}=2.8 \%$, bias $=90 \mathrm{mV})$, respectively. (e) Profile cut of the resolved topography along the green line in (c). The profile indicates the different areas of the sample (glass and $10 \mathrm{~nm}$-high gold lines), hidden in an overly low-estimated topography at the edges of the gold lines (indicated by red arrows). (f) Schematic representation of the origin of the overcompensation from the SICM controller. The black arrow indicates the increase in the magnitude of the ion flow out of the pipet as the lateral contact with the sample (represented by a red star) results in an increased working distance $d$ of the pore opening to the substrate than the setpoint distance (note: a flow of opposite ions also occurs to balance the charge but is not shown).

Figure 2(c) shows the SICM results of scanning with the nanopore probe ( $\mathrm{SP}=2.8 \%$ as earlier optimized) a patterned area of $90 \mathrm{~nm}$ wide gold lines of $300 \mathrm{~nm}$-pitch. The nanopore probe clearly identifies nanoscale features on the substrate unlike the apex probe [Fig. S3(c) of the supplementary material], but the resulting SICM topography is notably different from the true features geometry present on the substrate. Indeed, the gold lines are imaged as $136 \mathrm{~nm}$ wide lines, with a surprisingly low topography border on each side of the gold steps. A profile cut [Fig. 2(e)] indicates that the topography between the glass substrate and the gold line is resolved at a $10 \mathrm{~nm}$ height but gets hidden by a lateral border artefact imaged as a lower topography, down to $-15 \mathrm{~nm}$ relatively to the glass substrate (pointed by the red arrows), even reaching $-35 \mathrm{~nm}$ on other occurrences. The discrepancy between the true features geometry and the resulting SICM image can be explained by a strong convolution with the probe geometry as notified by the force feedback of Fig. 2(d). Indeed, the location of the high forces applied onto the sample corresponds with the topography dips. A possible interpretation is depicted in Fig. 2(f): the inability of the probe to sense side features leads to probe collision into the sample. The probe opening is prevented to get closer to the sample because of the sides of the probe contacting the sample: the larger probe opening-sample working distance creates a local current enhancement at the pore (represented by the weighted black arrow) that the SICM controller compensates by commanding the tip down. This leads to a topography consequently measured wrongly as low. Although this lateral border effect is strongly visible at the nanoscale, it actually also exists in the imaged microscale PDMS sample (profile cut of Fig. S4 of the supplemental material), but was overlooked from the high-aspect ratio of the features hiding the nanoscale topography variations associated with this effect. Thinner probes such as glass pipettes benefit from reduced lateral dimensions that enable probing closer to step features before such an effect occurs.

\section{AFM imaging with simultaneous current for SICM interpretation}

To test our hypothesis on the origin of the identified border effect, which is imaged too (i.e., overly) low with the SICM controller, we switched the FluidFM controller to act as an AFM, i.e., controlling the deflection instead of the ionic current to scan the sample. Such a strategy allows to observe the ionic current variations while following the topography, thanks to the force feedback.

Figure 3 shows the results of imaging in AFM contact mode the same $10 \mathrm{~nm}$ aspect-ratio gold lines as before but with variable lateral dimensions $(115 \mathrm{~nm}$ and $65 \mathrm{~nm}$, while the case of $90 \mathrm{~nm}$ is presented in Fig. S5 of the supplementary material). The force SP in contact mode is of $4 \mathrm{nN}$, while the related ion current flowing through the tip opening is simultaneously recorded. Figures 3(a) and 3(f) show that for both $115 \mathrm{~nm}$ and $65 \mathrm{~nm}$ gold dimensions, the nanopore probe is able to resolve the lines in the AFM topography image although with a noticeable probe convolution. For example, in the case of the $115 \mathrm{~nm}$ lines, the AFM topography profile cut of Fig. 3(d) evidences line features of $\sim 117$ nm but with slopes at the steps transition because of the pyramidal shape of the cantilever tip. Furthermore, the line topography height is only $6.4 \mathrm{~nm}$ as the tip cannot probe deep down to reach the underlying glass substrate between two gold lines. Interesting information is contained in the simultaneously recorded current image, where the gold lines are clearly identified too. Indeed, Fig. 3(b) reveals gold lines imaged as stripes of low current amplitudes, encompassed between high current amplitudes (lighter stripes). Those lighter stripes indicate the local current enhancement observed previously at the gold line borders. The glass substrate appears more (for the 65-nm lines case) or less (for the $115-\mathrm{nm}$ lines case) visible as a thin dark line, associated with the lower current amplitude attained when the probe gets as close as possible to the deeper glass substrate within the limits of the pyramidal shape of the probe. Figures 3(c) and 3(h) 
(a)Topography
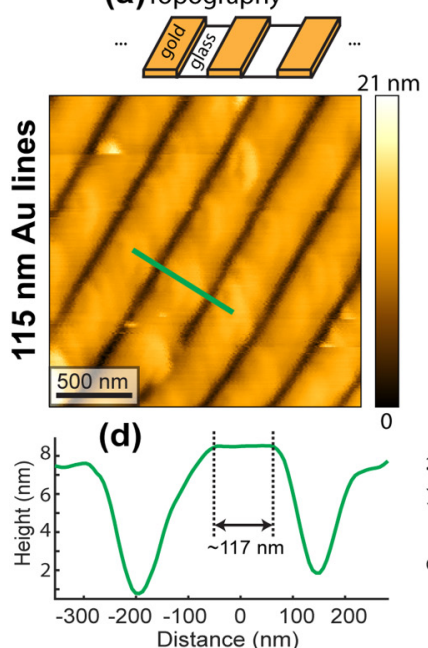

Distance (nm)

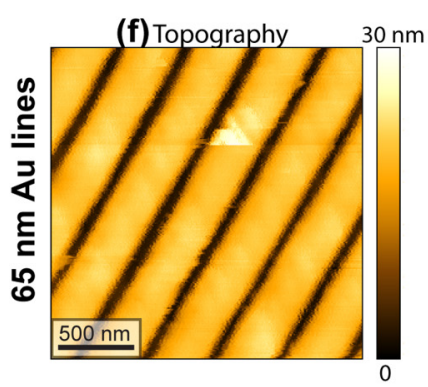

(b) Simultaneous current
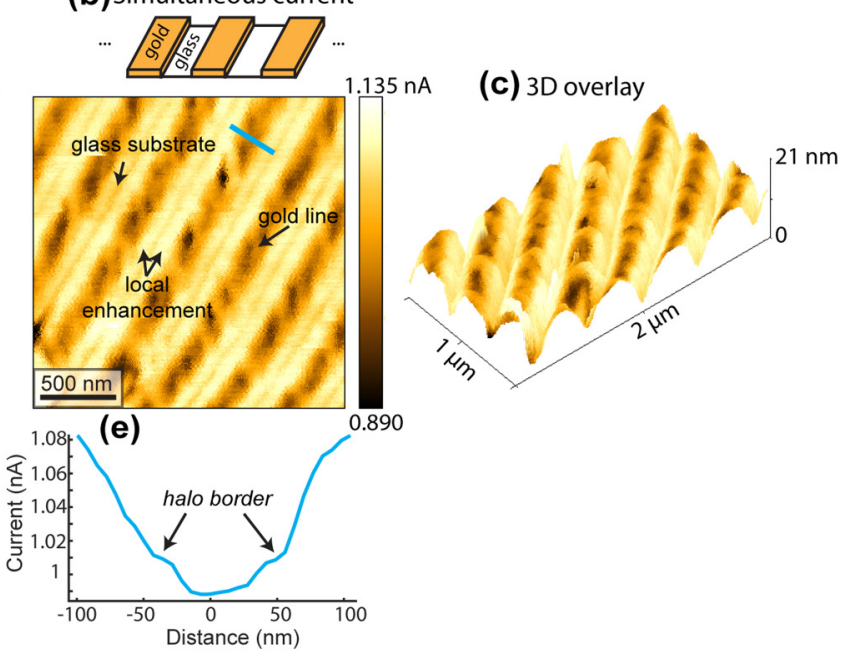

(g) Simultaneous current $1.11 \mathrm{nA}$

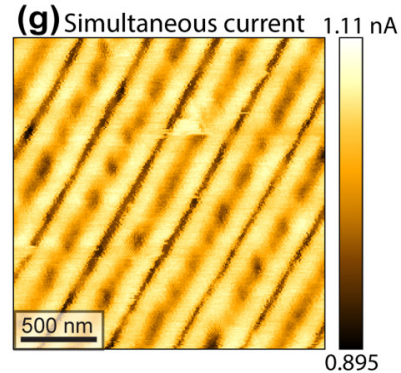

FIG. 3. (a) AFM topography and (b) simultaneous current when imaging $115 \mathrm{~nm}$-wide gold lines separated by $185 \mathrm{~nm} \quad(300 \mathrm{~nm}$ pitch $) \quad(\mathrm{SP}=4 \mathrm{nN}$, bias $=90 \mathrm{mV}$ ). (c) $3 \mathrm{D}$ representation of the imaged gold lines of [(a) and (b)] constructed by overlapping the simultaneous current color map onto the 3D relief given by the AFM topography. (d) Profile cut of the resolved topography along the green line of (a). (e) Profile cut of the recorded current along the blue line in (b). (f) AFM topography and (b) simultaneous current when imaging $65 \mathrm{~nm}$ wide-gold lines separated by $235 \mathrm{~nm}(\mathrm{SP}=4 \mathrm{nN}$, bias $=90 \mathrm{mV}$ ). (d) Corresponding 3D overlay built similarly to (c). show the 3D topography resolved by the AFM, while the simultaneous current is overlaid as a color map. The $3 \mathrm{D}$ images highlight the correlation between the lateral current enhancement and the opening angle of the probe that prevents closer working distances to the sample under limiting forces. This increased current is responsible for the SICM lateral border effect previously identified. Consequently, the wide opening angle of the nanopore tip is detrimental when performing nanoscale imaging as it leads to SICM topography artefacts visible as an overly low-estimated topography at the border of a step feature, along with high forces applied onto the sample.

However, in both the simultaneous current images of the AFM feedback [Figs. 3(b) and 3(g)] and in SICM mode [Fig. 2(c)], the nanopore probe is able to resolve, although with artefacts, the nanoscale gold lines down to at least 65 $\mathrm{nm}$ width, unlike the apex probe that cannot resolve the nanoscale features in either modes, even for the wider 115 $\mathrm{nm}$ lines (Section SI-3 of the supplementary material). On the contrary, the resolution of the nanopore probe is such that it even identifies the non-uniform distribution of the patterned gold lines on the glass substrate, imaged as discontinuous dark features [Figs. 3(b) and $3(\mathrm{~g})$ ]. This comes from gold islanding of the thin-deposited $10 \mathrm{~nm}$ gold layer that creates visible cracks in the gold patterns, as confirmed by SEM (Figs. S2(d) and S2(e) of the supplementary material).

A closer inspection of the images reveals that the gold features appear encompassed by a lighter halo border, further evidenced on the current profile of Fig. 3(e) of a $115 \mathrm{~nm}$ gold line: it depicts a $2.1 \%$ current increase at the feature border (at $1.009 \mathrm{nA}$ current amplitude) compared to its darker center $(0.988 \mathrm{nA})$, while it can reach up to $7.7 \%$ contrast on the darkest features. The $65 \mathrm{~nm}$ gold line of Fig. $3(\mathrm{~g})$ gives a lower but still existing contrast in current amplitude of $2.3 \%$ difference between the border and center of the darkest features. A finite-element method (FEM) model allows explaining this artefact.

\section{Halo border artefact}

FEM modeling offers a powerful contribution to the interpretation of SICM imaging: the simulation of the experimental data uncovers image formation processes and artefacts. $^{7,8,23}$

In this work, a 2D FEM model was built to interpret the recorded current images generated as the nanopore probe is scanned above a nanoscale feature. The geometry of the FluidFM nanopore probe was carefully reproduced from the geometrical estimations provided by SEM, HIM, and electrical resistance measurements discussed previously. The scanning working distance was estimated from geometrical considerations (Section SI-6 of the supplementary material) and set to $6 \mathrm{~nm}$ from the surface of the gold feature, simulated as a $10 \mathrm{~nm}$-high rectangle of varying width. Figure 4(a) shows the first $180 \mathrm{~nm}$ of the tip of a simulated FluidFM nanopore probe above a $115 \mathrm{~nm}$ wide feature, along with the calculated distribution of the ion current density under a 90 $\mathrm{mV}$ bias. As a comparison, Figure 4(b) depicts at same magnification the tip of a glass pipette above the $115 \mathrm{~nm}$ feature, with same $9 \mathrm{~nm}$ opening radius but with typical glass pipette lateral dimensions for high resolution (here $6.5 \mathrm{~nm}$ wall thickness, ${ }^{12}$ corresponding to $R \sim 1.7$ vs. 12 of the FluidFM) 
and half-opening angle $\left(2^{\circ}\right.$ vs. $\left.35.26^{\circ}\right)$. The calculated ion current density images of both probes confirm the important current squeezing happening under the tip walls ${ }^{7}$ (larger ion current density in the resistive gap below the walls). The higher current density on elongated lengths within the glass pipette compared to the rapidly dropping density of the FluidFM probe in the pyramid evidences the lower probe electrical resistance conferred by the increased opening angle of the FluidFM nanopore probe.

Figure 4(c) depicts the evolution of the probe current as the two tips are scanned laterally above a centered 10 $\mathrm{nm}$-high gold feature of varying dimensions $(115 \mathrm{~nm}, 90 \mathrm{~nm}$, and $65 \mathrm{~nm}$ as experimentally). The current is normalized to the current amplitude at $200 \mathrm{~nm}$ lateral distances from the feature. The simulated variations of the current when scanning the FluidFM probe above the features from left to right show similar qualitative results as experimentally, with lines imaged as a center dark feature bordered by a halo (for example, in the case of the $115 \mathrm{~nm}$ line, a $5.8 \%$ amplitude current increase is recorded at the side halo compared to the center), slowly disappearing toward a dip halo as the feature width is reduced [also observed experimentally in Fig. 3(g) for the $65 \mathrm{~nm}$ lines and Fig. S5(g) of the supplementary material].

The cartoons of Fig. 4(d) explain the halo artefact by looking at the path of the ion flow through the scanning probe opening depicted as weighted black arrows. When the probe sidewall comes above the feature, the squeezing of the current under the lateral sidewalls of the probe (depicted as thinner black arrows) contributes to a decrease in current due to the higher resistive path (i). When the probe gets centered above the feature, two scenarios are possible. When the probe opening is small enough compared to the feature size (ii), the increased resistive path below the opening and probe walls leads to further decrease in current: this effect results in imaging the feature with a center at low current amplitude circled by a higher current amplitude halo \{e.g., the $115 \mathrm{~nm}$ features observed experimentally [Fig. 3(b)] and in silico [Fig. 4(c)]\}. Second scenario: the probe opening is big relatively to the feature dimensions (iii), preventing enough overlapping area under the probe walls to constrain the ion flow under the tip (reduced resistive path). The consequent reduced resistive path for a feature below the tip opening (high amplitude) compared to under the probe walls (lower amplitude) leads to imaging the feature as an empty/dip halo [Fig. 4(c) $50 \mathrm{~nm}$ line width in silico and Fig. S5(g) of the supplementary material experimentally]. The empty halo effect (also referred to as "ring") had already been identified by Rheinlaender and Schäffer,"10 for sample features of dimensions below the tip opening. Here, we complement this study by evidencing that the identified ring effect is a particular case of the tip wall's impact in SICM imaging. It can occur also for feature sizes bigger than the probe opening [Fig. 4(c) and Fig. S5(g) of the supplementary material] but can also translate as a side halo bordering a central feature. The effect is a complementary situation to the halo artefact identified in silico by Edwards et al. ${ }^{11}$ in the case of a pit feature. The effect is from its origin dependent on the dimensions of the tip opening, probe side walls, feature size, and the working distance that will determine if the halo appears empty (dip) or full (with a central feature).

Importantly, although the impact of probe wall was evidenced by following the current variations in the FEM simulations and in the simultaneous current images acquired in AFM mode, it will exist similarly in an SICM image by appearing as a higher SICM topography where lower current amplitudes are recorded (as expected from the working principle of the SICM controller). For example, one can see how the $90 \mathrm{~nm}$ gold line of Fig. 2(e) was actually resolved as a central feature (peaking to a $10 \mathrm{~nm}$ height) bordered by two lower side features (reaching a $7 \mathrm{~nm}$ height), as predicted from the current image of Fig. S5(e) of (a) FluidFM nanopore

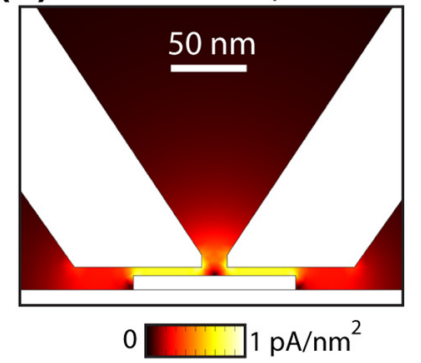

(d)

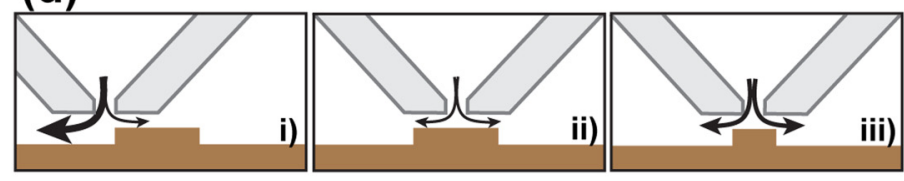

(b) glass pipette

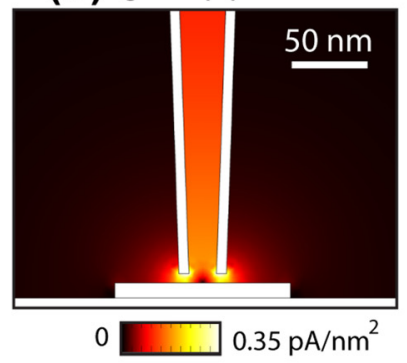

(c)

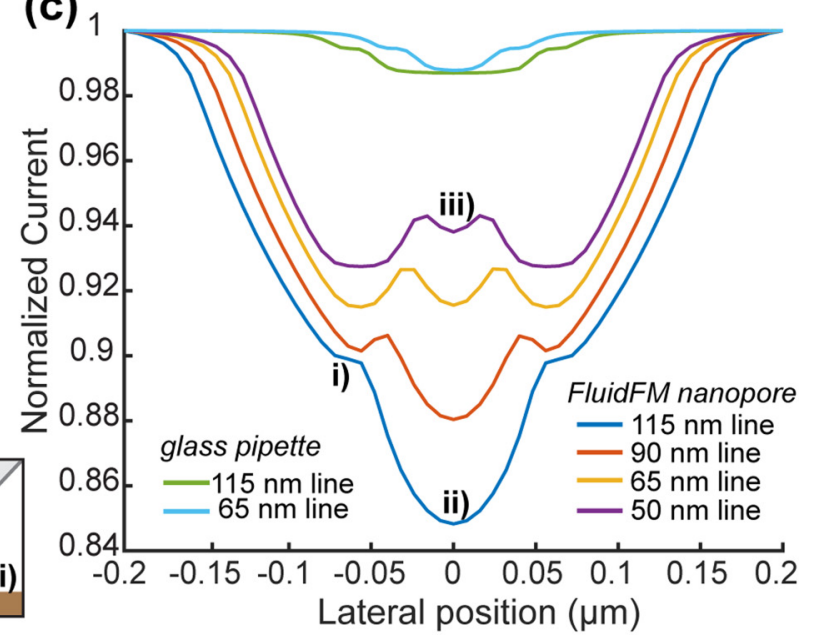

FIG. 4. [(a) and (b)] FEM-calculated distribution of the ion current density when the probe is centered above a $115 \mathrm{~nm}$ wide feature (probe working distance of $6 \mathrm{~nm}$ from the feature surface), at a $90 \mathrm{mV}$ applied bias. (c) Evolution of the simulated current when imaging a centered feature (at $0 \mu \mathrm{m}$ lateral position) of different widths (see legends) with a nanopore or a glass pipette probe (both $9 \mathrm{~nm}$ radius opening) at a constant distance (here $6 \mathrm{~nm}$ from the feature top surface). The lateral position represents the relative distance of the probe opening from the feature center. The current is normalized to the current amplitude 200 nm away from the feature. (d) Explanatory schematics of the ion flow as the probe is positioned above a feature. (i), (ii), and (iii) are associated with the positions identified in (c). The weight of the arrows indicates the relative magnitude of the ion flow out of the pipet, impacted by the presence of the sample features. 
the supplementary material imaging the $90 \mathrm{~nm}$ gold lines as a central dark feature bordered by a lighter halo.

The sidewall effect could be revealed in this work because of the design of a nanopore probe with consequent tip wall dimensions $(R \sim 12)$ via ion milling (FIB, HIM) customization. The nanopore geometry contrasts with conventional glass pipette geometries displaying a smaller wall thickness and opening angle. As an example, the numerical result of scanning $65 \mathrm{~nm}$ and $115 \mathrm{~nm}$ lines with a glass pipette of the same opening as the FluidFM nanopore is shown in Fig. 4(c) as a light blue and green trace, respectively. It evidences that the side wall effect with glass pipettes although existent is limited from their lower signal to noise ratio, attributed to reduced wall thicknesses and the smaller current amplitude variations offered by those probes (high electrical probe resistance associated with a limited opening angle). The effect could interestingly still be experienced computationally ${ }^{11}$ and experimentally ${ }^{10}$ in some works with glass pipettes as a small topography inflection. Therefore, FluidFM nanopore probes do display improved SICM sensitivity as expected from simulation and theoretical works but at the cost of an inherent sidewall effect on the imaged features that is no longer negligibly hidden but instead distorts the final image more evidently. By affecting the probe SICM sensitivity, the probe wall thickness and opening angle impact the final SICM resolution by influencing the signal to noise determining the detection of a feature. Additionally, the fact that an increasing wall thickness results in smearing the feature on bigger lateral distances [Fig. 4(c)] further evidences the contribution of the probe wall thickness in the quantification formula of the lateral resolution that can no longer only be limited to the inner probe radius. Further analysis with the support of the FEM model will help determining the relative contribution of the probe wall thickness in the quantification of the lateral resolution.

\section{CONCLUSION}

We successfully engineered a nanopore probe meeting geometry requirement identified from models and simulations to make SICM more sensitive to tip-sample separation. The nanopore diameter $(<20 \mathrm{~nm})$ coupled with an increased lateral thickness $(R \sim 12)$ and a wide pyramidal probe opening angle $\left(\sim 70^{\circ}\right.$ total angle) ensures higher current sensitivity to distance variations, as revealed from approach curves and FEM simulations. Combining the SICM nanopore opening with a FluidFM allows to fully investigate the implications of the new probe geometry in terms of SICM imaging artefacts and mechanical contacts measured by an additional force sensor. The forces reveal more than sample contacts, but also errors in the SICM feedback that could be interpreted by operating under AFM control to understand SICM image formation via the simultaneously recorded current. We show that the nanopore probe can image features down to the nanoscale ( $65 \mathrm{~nm}$ feature width here) which could not be resolved with bigger pores $(300 \mathrm{~nm}$ apex probes). Yet, the resulting SICM image topography from the nanopore is distorted because of the increased lateral dimensions of the probe (probe opening angle and wall thickness).
The probe lateral dimensions lead to local current enhancement on step edges resulting in imaging step borders with excessively low resolved SICM topography artefact. Whereas the effect can be left unnoticed when imaging high-aspect ratio features (at the cost of high forces applied onto the sample as recorded by the force feedback), this nanoscale effect gets more visible when probing nanoscale features, imaged with an altered topography. The higher SICM sensitivity of the probe allowed identifying the impact of the probe walls in the SICM imaging process because of the increased current amplitude variations the nanopore probe records. It especially revealed that the wall of the tip can result in imaging features with a dip or a bordering halo artefact, observed experimentally and studied via FEM simulations.

The side wall effect could play an important role in SICM imaging; therefore, care should be taken in limiting the wall dimensions of the probe when pushing toward highresolution. Recent works aiming to increase the resolution by using small nanopores (e.g., via local physical shrinking of the probe aperture ${ }^{24,25}$ or using biological pores in lipid membranes ${ }^{26,27}$ ) concomitantly result in the formation of probes with bigger walls, which will most likely face such side wall imaging effects introduced herein.

\section{ACKNOWLEDGMENTS}

This work was funded by the EUROSTARS project (Grant No. E!11644). The authors would like to thank Edin Sarajlic (SmartTip BV, The Netherlands), Patrick Frederix (Nanosurf AG, Switzerland), Pablo Dörig, and Dario Ossola (Cytosurge AG, Switzerland) for their support as well as Stephen Wheeler (ETH Zurich) and the personnel of the ETH ScopeM facility for technical assistance. M.A. acknowledges a Marie Skłodowska-Curie action (Project Reference: No. 706930). I.S. is thankful to Empa for financial support and to the Swiss National Science Foundation (SNF) for support in equipment procurement (REquip 206021_133823).

\section{SUPPLEMENTARY MATERIAL}

See supplementary material for the description of the FluidFM nanopore electrical resistance, optical and SEM images of the working samples, imaging results with a 300 nm FluidFM apex probe, profile cut of the PDMS topography resolved with the FluidFM nanopore, imaging results with a bigger nanopore opening, and estimation of the working distance of the probe aperture to the sample.

${ }^{1}$ P. K. Hansma, B. Drake, O. Marti, S. A. Gould, and C. B. Prater, Science 243, 641 (1989)

${ }^{2}$ Y. E. Korchev, C. L. Bashford, M. Milovanovic, and I. Vodyanoy, Biophys. J. 73, 653 (1997).

${ }^{3}$ F. Anariba, J. H. Anh, G.-E. Jung, N.-J. Cho, and S.-J. Cho, Mod. Phys. Lett. B 26, 1130003 (2012).

${ }^{4}$ G. Binnig, C. F. Quate, and C. Gerber, Phys. Rev. Lett. 56, 930 (1986).

${ }^{5}$ J. Rheinlaender, N. A. Geisse, R. Proksch, and T. E. Schäffer, Langmuir 27, 697 (2011).

${ }^{6}$ J. Seifert, J. Rheinlaender, P. Novak, Y. E. Korchev, and T. E. Schäffer, Langmuir 31, 6807 (2015).

${ }^{7}$ J. Rheinlaender and T. E. Schäffer, J. Appl. Phys. 105, 094905 (2009).

${ }^{8}$ S. Del Linz, E. Willman, M. Caldwell, D. Klenerman, A. Fernández, and G. Moss, Anal. Chem. 86, 2353 (2014). 
${ }^{9}$ A. E. Weber and L. A. Baker, J. Electrochem. Soc. 161, H924 (2014).

${ }^{10}$ J. Rheinlaender and T. E. Schäffer, Anal. Chem. 87, 7117 (2015).

${ }^{11}$ M. A. Edwards, C. G. Williams, A. L. Whitworth, and P. R. Unwin, Anal. Chem. 81, 4482 (2009).

${ }^{12}$ A. I. Shevchuk, G. I. Frolenkov, D. Sánchez, P. S. James, N. Freedman, M. J. Lab, R. Jones, D. Klenerman, and Y. E. Korchev, Angew. Chem. Int. Ed. Engl. 45, 2212 (2006).

${ }^{13}$ Y. E. Korchev, M. Milovanovic, C. L. Bashford, D. C. Bennett, E. V. Sviderskaya, I. Vodyanoy, and M. J. Lab, J. Microsc. 188, 17 (1997).

${ }^{14}$ A. Meister, M. Gabi, P. Behr, P. Studer, J. Vörös, P. Niedermann, J. Bitterli, J. Polesel-Maris, M. Liley, H. Heinzelmann, and T. Zambelli, Nano Lett. 9, 2501 (2009).

${ }^{15}$ O. Guillaume-Gentil, E. Potthoff, D. Ossola, C. M. Franz, T. Zambelli, and J. A. Vorholt, Trends Biotechnol. 32, 381 (2014).

${ }^{16}$ D. Ossola, L. Dorwling-Carter, H. Dermutz, P. Behr, J. Vörös, and T. Zambelli, Phys. Rev. Lett. 115, 238103 (2015).

${ }^{17}$ R. F. Tiefenauer, K. Tybrandt, M. Aramesh, and J. Vörös, ACS Nano 12 , 2514 (2018).
${ }^{18}$ R. F. Tiefenauer, T. Dalgaty, T. Keplinger, T. Tian, C. Shih, J. Vörös, and M. Aramesh, Small 14, 1801187 (2018).

${ }^{19}$ I. Shorubalko, L. Pillatsch, and I. Utke, Helium Ion Microscopy (Springer, 2016).

${ }^{20}$ M. Aramesh, Y. Mayamei, A. Wolff, and K. K. Ostrikov, Nat. Commun. 9, 835 (2018).

${ }^{21}$ I. Shorubalko, K. Choi, M. Stiefel, and H. G. Park, Beilstein J. Nanotechnol. 8, 682 (2017).

${ }^{22}$ D. Ossola, M.-Y. Amarouch, P. Behr, J. Vörös, H. Abriel, and T. Zambelli, Nano Lett. 15, 1743 (2015).

${ }^{23}$ D. Thatenhorst, J. Rheinlaender, T. E. Schäffer, I. D. Dietzel, and P. Happel, Anal. Chem. 86, 9838 (2014).

${ }^{24}$ L. J. Steinbock, J. F. Steinbock, and A. Radenovic, Nano Lett. 13, 1717 (2013).

${ }^{25}$ X. Xu, C. Li, Y. Zhou, and Y. Jin, ACS Sens. 2, 1452 (2017).

${ }^{26}$ Y. Zhou, L. K. Bright, W. Shi, C. A. Aspinwall, and L. A. Baker, Langmuir 30, 15351 (2014).

${ }^{27}$ F. C. Macazo and R. J. White, J. Am. Chem. Soc. 138, 2793 (2016). 